\title{
PERFIL CLÍNICO-EPIDEMIOLÓGICO DE CRIANÇAS E ADOLESCENTES QUE VIVEM E CONVIVEM COM ESPINHA BÍFIDA
}

\section{CLINICAL AND EPIDEMIOLOGICAL PROFILE OF CHILDREN AND ADOLESCENTS WHO LIVE WITH SPINA BIFIDA}

\author{
Maria Aparecida Munhoz Gaíva ${ }^{1}$ \\ Emanuelle Righetto Corrêa ${ }^{2}$ \\ Elisete Ap. Rubira do Espírito Santo ${ }^{3}$
}

Gaiva MAM; Corrêa ER; Espírito Santo EAR. Estudo das variáveis materno-infantis na espinha bífida. Rev. Bras. Cresc. e Desenv. Hum. 2011; 21(1): 99-110.

\section{Resumo:}

O objetivo é analisar as variáveis clínico-epidemiológicas de crianças e adolescentes portadoras de espinha bífida. Trata-se de um estudo descritivo, transversal, constituído por 41 crianças e adolescentes entre zero e vinte anos de idade. A coleta de dados foi realizada no período de janeiro a maio de 2006, mediante aplicação de um

formulário. Os resultados revelam que 32 (78\%) crianças e adolescentes possuíam mielomeningocele, 22 (54\%) eram do sexo masculino; 32 (78\%) nasceram de parto cesárea; 29 (71\%) nasceram a termo, 31 (77\%) nasceram com peso considerado adequado. A bexiga neurogênica, a hidrocefalia, os pés tortos congênitos e o intestino neurogênico foram as complicações mais frequentes entre a população estudada. A maioria, 31 (76\%) famílias, possuía renda mensal de até três salários mínimos; 32 (78\%) mães declararam que sua gravidez não foi planejada e 33 (80\%) mães afirmaram não ter feito uso de ácido fólico nos primeiros meses de gestação. O estudo aponta a necessidade de maiores investimentos por parte do poder público, considerando que essa é uma malformação que pode ser prevenida e que medidas podem ser implementadas na rede básica de saúde.

Palavras-chave: anomalias congênitas; disrafismo espinal; defeitos do tubo neural; crianças portadoras de deficiência; assistência de enfermagem.

\footnotetext{
Enfermeira. Professora Doutora da Faculdade de Enfermagem - Universidade Federal dede Mato Grosso.

Graduanda de enfermagem da Universidade Federal de Mato Grosso.

3 Enfermeira. Professora Mestre da Faculdade de Enfermagem - Universidade Federal de Mato Grosso. Doutoranda da Universidade Federal de São Paulo

Correspondência para: Maria Aparecida Munhoz Gaíva - Universidade Federal de Mato Grosso - E-mail: mamgaiva@yahoo.com.br

$\mathrm{O}$ artigo é resultante de um estudo de Iniciação Cientifica (CNPq), vinculado ao Projeto de Pesquisa: Crianças e adolescentes portadoras de Defeitos de Fechamento do Tubo Neural, do Grupo de Pesquisa Projeto Argos, UFMT. Recebeu auxilio do CNPq por meio de bolsa de Iniciação Cientifica.
} 


\begin{abstract}
:
The objective is clinical and epidemiological profile of children and adolescents who live with spina bifida. It is a descriptive study, cross-sectional, formed by 41 children and adolescents between zero and twenty years of age. The data collection was carried out from January to May, 2006, by applying a form. The results reveal that 32 (78\%) children and adolescents had myelomeningocele, 22 (54\%) were males; 32 (78\%) were born by cesarean section; 29 (71\%) were term-born children, 31 (77\%) were born with an adequate weight. The neurogenic bladder, the hydrocephalus, the congenital clubfeet and the neurogenic intestine were the most common complications among the population studied. The majority, 31 (76\%) families had a monthly income of up to three minimum salaries; 32 (78\%) mothers stated that their pregnancies were not planned and 33 (80\%) mothers declared that they had not used folic acid in the first months of pregnancy. The study indicates a need for greater investments by the governmental instances, considering that this is a malformation that can be prevented and that measures must be taken in the Basic Healthcare Network.
\end{abstract}

Key words: congenital anomalies; spinal dysrafism; neural tube defects; disabled children; nursing care.

\section{INTRODUÇÃO}

A falha em fechar uma porção do tubo neural, ou reabertura de uma região do tubo após o fechamento bem sucedido, pode levar a várias malformações. A mais comum delas é a Espinha Bífida que pode classificar-se como espinha bífida oculta ou espinha bífida cística ${ }^{1}$. A espinha bífida cística ou aberta apresenta-se como protusão sacular externa e as duas principais formas são a Mielomeningocele, que se refere à extensão do tecido do sistema nervoso central, associado a um déficit neurológico acentuado. Esse déficit ocorre porque o tecido nervoso é incorporado à parede do saco, prejudicando o desenvolvimento das fibras nervosas. Pode ocorrer em qualquer ponto da coluna vertebral, mas é mais comum nas regiões lombares e sacrais. Como resultado disso haverá sempre alguma paralisia e perda da sensação abaixo da região prejudicada².

A outra forma, a Meningocele é a menos comum das duas. Nesse tipo, usualmente os nervos não estão prejudicados e são capa- zes de funcionar, por isso pouca incapacidade pode estar presente nas pessoas com essa malformação ${ }^{1}$.

A espinha bífida oculta é a forma branda da espinha bífida e é muito comum; no entanto, nas pessoas afetadas, são poucos os problemas e ela associados. Frequentemente aparece tufo de pelos no local do defeito ${ }^{1}$.

Essa malformação possui herança multifatorial, ou seja, interação entre vários genes e fatores ambientais ${ }^{3,4,5}$. Dentre os fatores ambientais estão as condições socioeconômicas, deficiência de folato e alimentos contaminados com inseticidas. Ainda podem induzir à formação de tal defeito, a exposição materna a drogas antineoplásicas, anticonvulsivantes, agentes anestésicos e agentes infecciosos ${ }^{6,7}$.

Pessoas com essa malformação apresentam complicações que transformam essa doença em um sério problema de saúde pública, com repercussão na vida do indivíduo, família e sociedade ${ }^{4}$.

As crianças com meningocele ou mielomeningocele podem apresentar incapaci- 
dades crônicas graves, tais como: hidrocefalia, bexiga neurogênica, disfunção intestinal, problemas ortopédicos e paralisia de membros inferiores ${ }^{3,5}$. Além dessas incapacidades podem apresentar também transtornos emocionais sociais e psicossociais, e deficiência cognitiva.

A principal estratégia para a prevenção dos Defeitos de Fechamento do Tubo Neural (DFTN) para a mulher em idade reprodutiva é a promoção de um estilo de vida saudável, associado a uma nutrição adequada, com consumo de alimentos ricos em ácido fólico ${ }^{6}$. Estudos têm demonstrado que a suplementação periconcepcional do ácido fólico, três meses antes da gestação, até a décima segunda semana de gestação pode prevenir os DFTN ${ }^{6,9}$.

Os dados da incidência dos DFTN, especificamente da Espinha Bífida em nosso país são escassos. Dados estatísticos mais atuais mostram que a prevalência dos DFTN varia de $0,83 / 1000$ a $1,87 / 1000$ nascimentos $^{10}$.

No Brasil, os DFTN, entre eles a espinha bífida, assumem importante papel na morbidade e mortalidade infantis ${ }^{12,13}$. Além da morbimortalidade, esta malformação possui um caráter de cronicidade, com risco para o desenvolvimento de complicações clínicas que necessitam de tratamento contínuo e internações frequentes.

O convívio com crianças e adolescentes portadores de espinha bífida nos espaços da Associação de Espinha Bífida (AEB/MT) mostrou-nos a necessidade de realizar estudos para melhor conhecer quem é e come vive essa população em nosso Estado, dada a importância dessa malformação nos índices de mortalidade infantil e da demanda de cuidados de enfermagem, seja no âmbito hospitalar ou no cotidiano familiar.

A Espinha Bífida é uma condição crônica, pois seus portadores têm necessidade de cuidado profissional prolongado e continuado. As crianças com essa malformação demandam cuidados de enfermagem, tais como cateterismo vesical, administração de medica- mentos, prevenção de lesões de pele, dentre outros.

A disponibilidade de dados acerca dos portadores de espinha bífida em nosso Estado cria oportunidade para o aprofundamento da problemática das malformações congênitas, o que tem grande relevância na definição de estratégias políticas para a redução de sua incidência.

Desta maneira, o objetivo é analisar as variáveis clínico-epidemiológicas de crianças e adolescentes portadoras de espinha bífida.

\section{MÉTODO}

Trata-se de um estudo descritivo do tipo transversal de abordagem quantitativa. Como não existem dados numéricos sobre os portadores de Espinha Bífida em Mato Grosso, já que nem mesmo temos a prevalência dos DFTN, a única fonte de informações sobre esses portadores estão localizadas na Associação de Espinha Bífida de MT, por esse motivo foi considerada nossa fonte de dados.

Atualmente, todas as crianças com Espinha Bífida que nascem nos hospitais da capital são encaminhadas para a AEB/MT, ali suas famílias são inscritas e têm a oportunidade de conhecer melhor a malformação e o contexto da assistência de saúde que seus filhos irão necessitar. Por outro lado, como a maioria dos hospitais do interior do estado não possui as condições necessárias para o atendimento dessas crianças, elas são encaminhadas para tratamento na capital. Desse modo, praticamente todas as crianças com Espinha Bífida hoje em nosso estado estão inscritas e recebem apoio da AEB/MT.

A investigação foi realizada junto à AEB/ MT, uma entidade sem fins lucrativos, fundada em fevereiro de 2004 por pais de portadores de espinha bífida, sediada em Cuiabá-MT. AAEB/ MT contava no momento da realização do estudo com 71 associados, oriundos das várias ci- 
dades do estado de Mato Grosso, sendo que destes, 58 eram crianças e adolescentes.

Do total da população eleita para o estudo (58), 17 crianças/adolescentes não foram incluídas na amostra por dificuldades em obter os dados, uma vez que suas fichas de cadastro na AEB estavam incompletas e a maioria delas morava no interior do estado e não foi possível fazer contato com as famílias por via telefônica ou por correspondência. Assim, neste estudo, optamos pela amostra de conveniência, que se constituiu de 41 crianças e adolescentes portadores de espinha bífida, com idade entre zero a 20 anos de vida.

Os dados foram colhidos utilizando-se um instrumento desenvolvido pelas pesquisadoras, abrangendo variáveis relacionadas aos pais: idade, escolaridade, renda familiar, número de membros da família; variáveis referentes à gestação e parto: idade materna na ocasião da gestação, gravidez planejada, número de consultas no pré-natal, uso de medicações, álcool e fumo na gravidez, uso de ácido fólico, número de filhos vivos ou mortos em gestações anteriores, tipo de gravidez e tipo de parto. Em relação às crianças foram estudadas as seguintes variáveis: peso ao nascer, idade gestacional, tipo de espinha bífida, complicações e sequelas associadas, condições de saúde atual e tipo de acompanhamento de saúde, uso de autocateterismo vesical, uso de órtese e meios auxiliares de locomoção, atividades escolares e lazer. Essas variáveis foram definidas considerando o perfil epidemiológico dos DFTN/espinha bífida em nosso país. A coleta de dados foi realizada no período de janeiro a maio de 2006.

A aplicação do formulário foi dividida em dois momentos: primeiramente foram coletadas as informações de identificação e sociodemográficas das famílias, disponibilizadas nas fichas de cadastro/adesão da AEB/MT. No segundo momento, as famílias e crianças/ adolescentes foram contatadas pelas pesquisadoras nas reuniões mensais da AEB/MT, ou por correspondência e/ou telefone, para obter informações referentes às condições da gestação, parto e nascimento, assim como a saúde atual das crianças/adolescentes. Foram utilizados também relatórios médicos disponibilizados pelas famílias, para obter informações acerca da malformação e seu tratamento.

Os dados foram codificados e registrados em um banco de dados na planilha do Programa Excel versão Office 2000 da Microsoft e, posteriormente, agrupados para realização da análise descritiva pelo Programa SPSS versão 15.0.

O estudo foi desenvolvido dentro dos padrões éticos de pesquisa envolvendo seres humanos estabelecidos pela Resolução nº. 196/ 96 do Conselho Nacional de Saúde, tendo sido aprovado pelo Comitê de Ética em Pesquisa do Hospital Universitário Júlio Muller, parecer $n^{\circ}$ 203/2005. As famílias/mães, que concordaram com a participação do filho no estudo, receberam informações detalhadas sobre sua finalidade e objetivos e assinaram o Termo de Consentimento Livre e Esclarecido (TCLE), garantindo-se o anonimato e assegurando-se a liberdade de desistir a qualquer momento.

\section{RESULTADOS}

Em relação ao sexo foi observada uma distribuição praticamente equivalente, com 22 (54\%) crianças/adolescentes do sexo masculino e 19 (46\%) do sexo feminino, com idade média de 8,3 anos, a menor idade encontrada foi de sete meses e a maior idade foi 20 anos. Em relação ao local de residência, 24 (59\%) crianças/adolescentes moravam em Cuiabá e 17 (41\%) no interior do estado.

Observou-se um elevado percentual de crianças que nasceram com peso considerado adequado, ou seja, acima de $2500 \mathrm{~kg}$ (Tabela 1). A média de peso foi de $3,06 \mathrm{~kg}$. O menor peso de nascimento encontrado foi de $1500 \mathrm{~kg}$ e o maior 4800 kg. Quanto à idade ges7tacional, 
Tabela 1: Distribuição das crianças/adolescentes estudados segundo variáveis referentes às condições de nascimento, cor, idade atual e sexo.

\begin{tabular}{|c|c|c|}
\hline \multirow[t]{2}{*}{ Variável } & \multicolumn{2}{|c|}{ Distribuição } \\
\hline & $N^{o}$ & $\%$ \\
\hline \multicolumn{3}{|l|}{ Idade } \\
\hline $0-4$ anos & 09 & 22 \\
\hline 5-9 anos & 13 & 32 \\
\hline 10-14 anos & 13 & 32 \\
\hline 15-20 anos & 06 & 14 \\
\hline \multicolumn{3}{|c|}{ Média e DP: 8,3 \pm 5,4 } \\
\hline \multicolumn{3}{|c|}{ Min-Máx 7m-20 anos } \\
\hline \multicolumn{3}{|l|}{$\overline{\text { Sexo }}$} \\
\hline Feminino & 19 & 46 \\
\hline Masculino & 22 & 54 \\
\hline \multicolumn{3}{|c|}{ Peso ao nascer (gramas)* } \\
\hline$<2500 \mathrm{~g}$ & 03 & 7,4 \\
\hline 2500 g a 3999 g & 32 & 78 \\
\hline$>4000 \mathrm{~g}$ & 03 & 7,3 \\
\hline Sem informações & 03 & 7,3 \\
\hline \multicolumn{3}{|c|}{ Média e DP: 3,06 $\pm 0,65$} \\
\hline \multicolumn{3}{|c|}{ Min-Máx: $1.500-4.800$ g } \\
\hline \multicolumn{3}{|c|}{ Idade gestacional } \\
\hline$<37$ semanas & 12 & 30 \\
\hline e”37 semanas & 29 & 70 \\
\hline \multicolumn{3}{|l|}{ Cor } \\
\hline Branca & 24 & 59 \\
\hline Parda & 16 & 39 \\
\hline Negra & 01 & 2 \\
\hline
\end{tabular}

* Faixas de peso padronizadas pela Organização Mundial de Saúde e Organização Pan-americana de Saúde.

29 (71\%) delas nasceu a termo e 12 (29\%) prétermo. Observou-se a predominância de cor branca entre os sujeitos estudados.

Nas variáveis maternas referentes a gestação e parto (Tabela 2), a maioria (78\%) das mães declarou que sua gravidez não foi planejada e apenas uma mãe (2\%) referiu não ter realizado consultas de pré-natal, enquanto 27 (66\%) realizaram de seis a dez consultas prénatais. A maioria dos partos foi cesárea e gestação única. A gestação gemelar ocorreu uma vez, sendo que somente um dos gêmeos foi acometido pela malformação.
Tabela 2: Distribuição de variáveis maternas referentes à gestação e parto da população estudada.

\begin{tabular}{lcc}
\hline Variável & \multicolumn{2}{c}{ Distribuição } \\
& $\boldsymbol{N}^{\boldsymbol{o}}$ & $\boldsymbol{\%}$ \\
\hline Gravidez planejada & 9 & 22 \\
Sim & 32 & 32 \\
Não & & 32 \\
\hline Pré-natal & 01 & \\
Nenhuma consulta & 04 & 14 \\
$<6$ consultas & 27 & 46 \\
6 a 10 consultas & 07 & 54 \\
$>$ 10 consultas & 02 & 7,4 \\
Sem informações & & \\
\hline Uso de ácido fólico & 04 & 78 \\
Sim & 33 & 7,3 \\
Não & 04 & 7,3 \\
Não sabe informar & & \\
Tipo de parto & 08 & 30 \\
Normal & 32 & 70 \\
Cesárea & 01 & 59 \\
Fórceps & & 39 \\
Tipo de gravidez & 40 & \\
Única & 01 & \\
Dupla & & \\
\hline
\end{tabular}

A tabela 3 evidencia que a maioria das mães possuía entre 20 a 30 anos (59\%), por ocasião da gravidez do filho malformado, a menor idade foi 14 anos, enquanto a maior, 40 anos. Quanto à ocorrência de espinha bífida na primeira gestação foram registrados 12 casos (29\%), na segunda foram 14 (34\%) e o restante das crianças nasceu da terceira ou posteriores gestações. O passado obstétrico das mães mostrou a ocorrência de 11 casos de abortos espontâneos prévios. Constatou-se também que apenas oito (20\%) das famílias possuíam malformados na família.

Apenas quatro (10\%) mães declararam ter feito uso de álcool (socialmente) e sete (17\%) delas fizeram uso de cigarro (menos de uma carteira/dia) durante o período que antecedeu à concepção ou até o primeiro trimestre da gravidez. No que se refere ao uso de medi- 
Tabela 4: Características clínicas e saúde atual das crianças/adolescentes estudados.

\begin{tabular}{lcc}
\hline Variável & \multicolumn{2}{c}{ Distribuição } \\
& $\boldsymbol{N}^{\boldsymbol{o}}$ & \% \\
\hline Tipo de Espinha Bífida & & \\
Meningocele & 04 & 10 \\
Mielomeningocele & 32 & 78 \\
Espinha Bífida oculta & 01 & 2 \\
Não especificado & 04 & 10 \\
\hline Complicações/sequelas* & & \\
Hidrocefalia & 30 & 73 \\
Pés tortos & 29 & 70 \\
Bexiga neurogênica & 26 & 63 \\
\hline Tipo acompanhamento* & & \\
Neurologia & 34 & 83 \\
Ortopedia & 21 & 51 \\
Fisioterapia & 26 & 63 \\
Nefrologia & 18 & 44 \\
Urologia & 12 & 30 \\
\hline Acesso serviços saúde & & \\
Usuários do SUS & 16 & \\
Usuários convênios/planos & & 39 \\
de saúde & 25 & 61 \\
\hline Uso de órteses* & & \\
Uso de talas, tutor longo, & & \\
bota, colete, fixador, etc & 22 & 53 \\
Uso de cadeira rodas & 26 & 63 \\
Uso de muleta e andadores & 22 & 53 \\
\hline Uso de Cateterismo & & \\
intermitente & & \\
Sim & & \\
Não & & \\
\hline Tratamento em outros & & \\
centros & & \\
Sim & & \\
Não & & \\
Uso de medicamentos & & \\
Sim & & \\
Não & & \\
\hline & & \\
\hline
\end{tabular}

* Algumas crianças/adolescentes possuíam mais de uma complicação/sequelas e eram companhadas por vários profissionais e faziam uso de mais de uma órtese ou equipamento para locomover. psicólogos. Dentre as 41 crianças/adolescentes, 16 (39\%) faziam acompanhamento em outros serviços de saúde fora do Estado.

Quanto ao acesso aos serviços de saúde, 25 (61\%) crianças/adolescentes eram usuários de convênios/planos de saúde e 16 (39\%) eram assistidos pelo Sistema Único de Saúde (SUS).

Foi observado que 22 (54\%) crianças/ adolescentes necessitavam de órteses, como tala, tutor longo, bota, colete e fixador; 22 (54\%) utilizavam equipamentos para locomoção (muletas e andadores/parapódio) e 26 (63\%) faziam uso de cadeira de rodas (Tabela 4).

No que se refere ao uso rotineiro de medicamentos, 29 (71\%) referiram usá-los atualmente. Dentre as classes terapêuticas mais utilizadas se encontram os antiespasmódicos vesicais, usados por 16 (39\%) crianças/adolescentes, os antiepiléticos, 10 (24\%), os antibióticos, 15 (36,5\%), os laxantes, quatro (10\%) e outros medicamentos como os anticolinérgicos, os antifiséticos e os analgésicos usados por cinco (12\%) dos participantes.

Entre as crianças/adolescentes estudados, 26 (63\%) faziam uso diário de sonda vesical, sendo que a maioria deles executava a sondagem intermitente quatro vezes ao dia.

Quanto à vida cotidiana, do total de crianças em idade escolar, cinco não frequentavam a escola; das 27 crianças que estavam estudando, 21 eram matriculadas na rede pública de ensino e nenhuma das crianças/adolescentes trabalhava.

Quanto às atividades de lazer realizadas pelas crianças/adolescentes, as mais citadas foram: assistir TV, brincadeiras em geral, ouvir música, fazer natação/brincar na piscina, jogar vídeo game, desenhar e pintar, ir à igreja e equitação terapêutica.

\section{DISCUSSÃO}

Quanto à distribuição da espinha bífida segundo o sexo, vários estudos demonstram 
maior frequência em recém-nascidos do sexo feminino ${ }^{11,12,13}$. No entanto, neste estudo a frequência maior foi do sexo masculino, o que é corroborado por outros estudos ${ }^{3,10}$.

Este estudo não mostrou relação entre o peso de nascimento e a espinha bífida, já que a maioria tinha peso maior que $2500 \mathrm{~g}$ ao nascer. Valores semelhantes foram encontrados em outras pesquisas ${ }^{11,13,14}$.

A relação entre etnia e mielomeningocele é descrita na literatura, como sendo mais comum entre brancos ${ }^{1,7}$. Estudo realizado em um hospital universitário de Curitiba (PR), ao analisar 31 crianças com mielomeningocele nascidas entre 1990 a 2000, encontrou uma preponderância de crianças brancas, mas relaciona essa característica à distribuição da população local ${ }^{15}$. Pesquisa realizada com uma população americana reafirma essa relação ao encontrar uma prevalência maior de DFTN em brancos do que em negros ${ }^{16}$. Neste levantamento também foi encontrada uma predominância entre brancos, o que não reflete a população residente em Mato Grosso, já que, de acordo com a Pesquisa Nacional por Amostra de Domicílios, realizada no ano de 2004, predominava no estado a cor parda (53\%), seguida pela branca com 38\% e 6,0\% da cor ou raça preta ${ }^{17}$.

Estudo Colaborativo Latino-Americano de Malformações Congênitas (ECLAMC) tem identificado, nos países em desenvolvimento, a gravidez não planejada como um fator de risco para malformações congênitas ${ }^{6}$. No presente estudo, a maioria, $78 \%$ das mulheres, não planejou a gravidez em que nasceu o filho com a malformação.

Quanto ao acompanhamento pré-natal, os resultados mostram que 27 (66\%) mães realizaram de seis a 10 consultas, considerado como adequado. Achado semelhante foi encontrado em estudo realizado no Distrito Federal ${ }^{14}$ e em Recife, no Instituto Materno Infantil de Pernambuco ${ }^{10}$.

A carência de ácido fólico é o fator de risco para os DFTN mais importante encontra- do até hoje e a suplementação de ácido fólico periconcepcional pode reduzir tanto a ocorrência como o risco de recorrência dessas malformações em torno de $70 \%^{3,18}$.

Neste levantamento, 80\% (33) das mães relataram não ter feito uso de ácido fólico na época da concepção e/ou durante o primeiro trimestre de gestação. Cabe destacar que não existe estudos sobre o uso do ácido fólico durante a gravidez com a população de gestantes em geral, em nossa região, o que nos impossibilitou destacar se existem diferenças entre as duas populações. Resultado semelhante foi observado em outro estudo realizado no Brasil, mostrando que nenhuma das mães de recém-nascidos com mielomeningocele estudados fez uso de ácido fólico para prevenção de DFTN ${ }^{15}$. Estudo realizado em maternidade universitária do Chile comparou as taxas de prevalência de DFTN antes e após a fortificação da farinha de trigo com ácido fólico no país, e os resultados mostraram um declínio significante dos casos de espinha bífida e anencefalia após a implementação dessa medida ${ }^{9}$.

Com o objetivo de diminuir o número de recém-nascidos que apresentam problemas de fechamento do tubo neural, o Ministério da Saúde do Brasil publicou a Portaria $n^{\circ} 710$ / 1999 e a Resolução 344/2002, com estratégias para aumentar a ingestão de ácido fólico, pela população, especialmente para as mulheres em idade fértil, regulamentando a adição de, no mínimo, 4,2 miligramas de ferro e 150 microgramas de ácido fólico para cada 100 gramas de farinha de milho e de trigo ${ }^{19,20}$. É bom salientar que a maioria dos participantes do estudo nasceu antes da implementação dessa medida governamental e que não há ainda nenhum estudo comparando as faixas de prevalência dos DFTN antes e após a fortificação das farinhas.

Foi observado que a grande maioria das crianças/adolescentes com espinha bífida nasceu por parto cesariano (78\%). Em 2008, mais 
da metade dos nascimentos vivos de mães residentes em Cuiabá, capital de Mato Grosso, foram de parto cesarianos $(59,9 \%)^{21}$. Estudo que identificou a prevalência de DFTN em uma maternidade de referência para gestação de alto risco no país mostrou que a maioria (68,5\%) das crianças nasceu de parto cesariano ${ }^{10}$. $\mathrm{O}$ parto cesáreo para fetos com espinha bífida é indicado pelo risco de ruptura da lesão, colaborando para a ocorrência de infecção e comprometimento neurológico.

Foi observado neste estudo que a maioria dos pais (56\%) e grande parte das mães (39\%) tinham baixo grau de escolaridade. O baixo nível de escolaridade e socioeconômico dos pais é descrito pela literatura como fator de risco para os DFTN ${ }^{22,24,25}$. Estudo realizado no Sul do Brasil ao analisar a frequência de recém-nascidos portadores de espinha bífida e os fatores associados a sua ocorrência evidenciou que a maioria das mães e pais dessas crianças tinha baixo grau de instrução ${ }^{13}$.

Os DFTN são considerados desordens multifatoriais, decorrentes da associação de fatores genéticos e ambientais, bem como a deficiência de folatos, dentre outros mecanismos que vêm sendo descritos na literatura.

A deficiência do ácido fólico considerado atualmente o fator de risco mais importante na etiologia dos DFTN foi durante muito tempo associado ao baixo nível socioeconômico da população. No entanto, para os autores, o hábito alimentar brasileiro do uso frequente de feijão e carne, pode favorecer a adequada ingestão de folatos na gestação, diferentemente do observado em populações dos EUA e Alemanha. Estudo realizado com gestantes de uma maternidade pública do Rio de Janeiro mostrou que aquelas com renda per capita abaixo da linha de pobreza tiveram um aumento importante na ingestão de folatos, comparativamente àquelas gestantes com renda maior de um salário mínimo 24.

A ordem de nascimento é outra variável que tem sido associada à ocorrência dos DFTN 5 . Referente à ordem de nascimento das crian- ças/adolescentes, observou-se que 14 (34\%) eram segundo filho e 12 (29\%) o primeiro. As pesquisas com população portadora de DFTN, entre eles a espinha bífida, têm mostrado resultados conflitantes em relação a variável paridade. Enquanto alguns estudos mostram que o risco para os DFTN aumenta com a multiparidade, três ou mais gestações ${ }^{5,12}$, outros estudos, inclusive com a população brasileira, descrevem uma menor prevalência de DFTN em multíparas ${ }^{3,13}$.

Neste estudo, 24 (59\%) mães possuíam entre 20 e 30 anos quando engravidaram do filho com espinha bífida, o que vai ao encontro dos achados de outros estudos, os quais mostram que as mães jovens/adolescentes e mães com 35 anos ou mais são mais propensas a ter um filho com DFTN ${ }^{6,23}$. No entanto, estudos realizados no país não encontraram relação entre a idade materna e a ocorrência de DFTN ${ }^{10,13}$, evidenciando que a relação entre idade materna e a ocorrência de DFTN é controversa.

A ocorrência de abortos espontâneos nas gestações anteriores é considerada como um fator de risco para os DFTN ${ }^{7}$. A análise do passado obstétrico das mães das crianças/adolescentes estudadas mostra que cinco (13\%) tiveram pelo menos um aborto espontâneo em gestações anteriores. Não obstante, há relato de pesquisa que não encontrou relação entre aborto espontâneo prévio e a ocorrência de espinha bífida ${ }^{13}$. Como não dispomos do percentual de abortos espontâneos da população em geral da região, não é possível afirmar que esse número é alto ou aceitável.

Quanto ao tipo de espinha bífida, destaca-se uma presença maior da mielomeningocele nas crianças/adolescentes estudados (78\%), o que é compatível com a literatura científi$\mathrm{ca}^{3,14,25}$. Cabe destacar que a informação do diagnóstico do tipo de DFTN, apesar de muitos deles terem sido colhidos em mais de uma fonte (formulários da AEB, informações das mães e relatórios médicos), pode não ter sido fidedigna em todos os casos. 
Neste estudo, as complicações mais encontradas entre a população estudada foram: bexiga neurogênica, hidrocefalia e pés tortos congênitos. A bexiga neurogênica é uma disfunção vesical secundária a um comprometimento do sistema nervoso, tendo como causa mais frequente a mielomeningocele ${ }^{26}$. Do total de 41 crianças/adolescentes estudados, 26 (63\%) tinham bexiga neurogênica. Estudo realizado em um hospital universitário brasileiro, com 31 crianças com mielomeningocele, 12 $(38,7 \%)$ delas teve o diagnóstico de bexiga neurogênica ${ }^{15}$. Resultado semelhante foi encontrado em pesquisa com uma amostra de 355 recém-nascidos com DFTN admitidos no Hospital de Base do Distrito Federal, destes, 102 (33,7\%) possuíam bexiga neurogênica ${ }^{14}$.

Ainda em relação às complicações da espinha bífida, 30 (73\%) das crianças estudadas tinham relato de tratamento de hidrocefalia. Essa é uma complicação muito frequente nessa malformação, a exemplo de pesquisa realizada em nosso país, que em uma população de 276 recém-nascidos com mielomeningocele, $90 \%$ apresentaram hidrocefalia ${ }^{15}$. Outros autores mostram que frequentemente a hidrocefalia está associada aos DFTN, principalmente as mielomeningoceles ${ }^{12,15,25}$. A presença de 29 (70\%) crianças/adolescentes com pés tortos congênitos em nossa amostra é concordante com o descrito em alguns estudos ${ }^{14,15}$.

Foi verificado que a maioria das crianças/adolescentes com espinha bífida deste estudo necessita de tecnologia assistiva, tais como: órteses, tutor, botas, palmilhas e outros meios auxiliares de locomoção como cadeira de rodas, andador e bengalas. Além disso, a maior parte delas faz uso cotidiano de sonda vesical e diversos medicamentos. Também necessitam de vários especialistas da área da saúde para atendêlos nas suas diferentes limitações, gerando um elevado custo as suas famílias e ao Estado.

A criança com espinha bífida possui alterações funcionais permanentes, embora muitas delas possam ser corrigidas ou amenizadas com o uso de órtese, exigindo do portador e de sua família mecanismos de adaptação, no decorrer das diferentes etapas de desenvolvimento, para que a criança cresça e se desenvolva desfrutando de qualidade de vida.

Estudo realizado com familiares de crianças com espinha bífida com idade entre 13 meses e seis anos, moradoras da cidade de Cuiabá, MT, com o objetivo de descrever a experiência das famílias quanto ao cuidado cotidiano dessas crianças, evidenciou que a família se vê sobrecarregada, pois vivencia experiências de cuidado que nunca desempenhou, na maioria das vezes, sem contar com o apoio de profissionais e serviços de saúde. Nesse processo de cuidar, a família tem que conviver com situações que a sociedade estigmatiza, como deficiência motora e uso de tecnologias assistivas, incontinência urinária e fecal. As famílias enfrentam também outras dificuldades para prover um cuidado de qualidade ao filho com espinha bífida, dentre elas, a questão financeira para atender às necessidades de saúde e reabilitação dos filhos ${ }^{27}$.

As crianças com espinha bífida precisam de tratamento e/ou acompanhamento multiprofissional ao longo de toda sua vida. No Mato Grosso, a extensão geográfica, a organização da atenção à saúde, as dificuldades de acesso a esses serviços e a deficiência do sistema público de saúde no atendimento às condições crônicas são fatores que impõem maiores desafios aos profissionais e às pessoas com malformações congênitas como a Espinha Bífida. Nesse sentido, a cidadania organizada coletivamente, por meio de redes de apoio como as associações, possibilita relações de trocas, obrigações recíprocas, além de ser uma ferramenta de luta na busca pelo acesso à assistência à saúde e melhor qualidade de vida.

No Brasil não estão disponíveis dados sobre o impacto e custos gerados pelos DFTN, mas sabemos que são patologias limitantes, geradoras de sofrimento e às vezes com sobrevida prolongadas e com ônus elevado para os familiares e para o Estado. 


\section{REFERÊNCIAS}

1. Moore KL, Persaud TVN. Embriologia clinica. $6^{a}$ ed. Rio de Janeiro: Guanabara Koogan; 2000.

2. Northrup H, Volcik KA. Spina bifida and other neural tube defects. Curr Probl Pediatr 2000; 30(10): 313-32.

3. Aguiar MJB. et al. Defeitos de fechamento do tubo neural e fatores associados em recém-nascidos vivos e natimortos. J. Pediatr 2003; 79(2):129-34.

4. Horovitz DDG, Llerena Junior JC, Mattos RA. Atenção aos defeitos congênitos no Brasil: panorama atual. Cad. Saúde Pública 2005; 21(4): 1055-1064.

5. Vieira AR, Taucher SC. Edad materna y defectos del tubo neural: evidencia para um efecto mayor em espina bífida que anencefalia. Rev. Méd. Chile 2005; 133: 62-70.

6. Cortés, MF. Prevención primaria de los defectos de cierre del tubo neural. Rev. Chil. Pediatr 2003; 74 (2):208-12.

7. Ogata AJ, Camano L, Brunoni D. Perinatal factors associated with neural tube defects (anancephaly, spina bifida and encephalocele) Rev. Paul. Med. 1992;110(4):147-151.

8. Ostos $\mathrm{H}$ et al. Disminución de la incidência de defectos de cierre del tubo neural em el Hospital Universitario de Neiva: posible efecto de la promoción del consumo de ácido fólico. Biomédica 2000; 20: 18-24.

9. Nazer HJ et al. Efecto de la fortificación de al harina con acido fólico sobre la evolución de las tasas de prevalencia al nacimento de malformaciones congénitas en los hospitales chilenos del ECLAMC. Rev. Méd. Chile 2007; 135(2): 198-204.

10. Pacheco SS et al. Prevalência dos defeitos de fechamento do tubo neural em recémnascidos do Centro de Atenção à Mulher do Instituto Materno Infantil Prof. Fernando Figueira, IMIP: 2000-2004. Rev.
Bras. Saúde Matern. Infantil 2006; 6 (supl 1): 535-542.

11. Furtado GJRD. Mielomeningocele: pacientes tratados cirurgicamente no Instituto Materno-Infantil de Pernambuco no ano de 1998. [dissertação mestrado]. Recife: Instituto Materno-Infantil de Pernambuco, Universidade Federal de Pernambuco; 2000.

12. Nazer HJ, López Camelo JS, Castilla EE. ECLAMC: Estudio de 30 años de vigilancia epidemiológica de defectos de tubo neural en Chile y en Latino América. Rev. Méd. Chile 2001; 129(5): 531-9.

13. Cunha CJ, Fontana T, Garcias GL, Martino-Roth MG. Fatores genéticos e ambientais associados a espinha bífida. Ver. Bras. Ginecol. Obstet. 2005; 27(5): 268-74.

14. Filgueiras MGC. Prevalência de recémnascidos portadores de Defeitos de Fechamento do Tubo Neural no Hospital de Base do Distrito Federal, 1993/2003. [dissertação mestrado]. Brasília: Universidade de Brasília; 2000.

15. Ulsenheimer MM. et al . Myelomeningocele: a Brazilian University Hospital experience. Arq. Neuro-Psiquiatr. 2004; 62(4): 963-968.

16. Stevenson RL et al. Decline in prevalence of neural tube defects in a high-risk region of the United States. Pediatrics 2000; 106(4): 677-83.

17. Instituto Brasileiro de Geografia e Estatística. Estados: Mato Grosso- Pnad 2004. Disponível em: http:// www.ibge.gov.br. [Acesso em 14 agost 2006].

18. Souza ASR, Arruda TAM, Amorim MMR, Pacheco AJC. Espinha Bífida: conceitos atuais. Femina 2007; 35(7): 455-62.

19. Ministério da Saúde (BR). Portaria $n^{\circ} 710$, de 10 de junho de 1999: aprova a Política Nacional de Alimentação e Nutrição. Diário Oficial da União [DOU] 1999 jun 11. 
20. Agência Nacional de Vigilância Sanitária (BR). Legislação em vigilância sanitária. Resolução RDC $n^{\circ}$ 344, de 13 de dezembro de 20002: aprova o regulamento técnico para a fortificação das farinhas de trigo e das farinhas de milho com ferro e acido fólico. Diário Oficial da União (DOU) 2002 dez 13.

21. Cuiabá. Secretaria Municipal de Saúde. Relatório de Gestão 2008. Diretoria de Planejamento. Cuiabá: 2009.

22. Schuler-Faccini LS, Leite JCL, Sanseverino MTV, Peres RM. Avaliação de teratógenos potenciais na população brasileira. Ciência \& Saúde Coletiva 2002; 7(1): 65-71.

23. Farley TF, Hambidge SJ, Daley MF. Association of low maternal education with neural tube defects in Colorado, 1989-1998. Public Health 2002; 116(2): 89-94.
24. Fonseca VM, Sichieri R, Basilio L, Ribeiro LVC. Consumo de folato em gestantes de um hospital público do Rio de Janeiro. Rev. Bras. Epidemiol. 2003; 6(4): 319-327.

25. Pereira ELR et al. Epidemiologia de pacientes com malformação de Chiari II internados no Hospital Fundação Santa Casa de Misericórdia do Pará. Rev. Pará. Med.2007; 21(2): 13-17.

26. Furlan MFFM. Cuidar de crianças portadoras de bexiga neurogênica: representações sociais das necessidades dessas crianças e suas mães. [dissertação mestrado]. Ribeirão Preto: Escola de Enfermagem de Ribeirão Preto Universidade de São Paulo; 1998.

27. Gaíva MAM, Neves, AQ, Siqueira FMG. O cuidado da criança com espinha bífida pela família no domicilio. Esc. Anna Nery Rev. Enferm. 2009; 13 (4):717-25.

Recebido em: 15/abr./09 Modificado em: 26/ago./10 Aceito em: 10/dez./10 